\title{
RELAÇÃO ENERGÉTICA E EMISSÃO DE GEE DO BIODIESEL DE PINHÃO MANSO: ANÁLISE SOB UMA PERSPECTIVA DINÂMICA
}

\author{
E.L.F.Ribeiro $^{1^{*}}$; H.L.Maranduba ${ }^{2}$; L.B.Oliveira ${ }^{2}$; J.A. de Almeida Neto ${ }^{3}$ e F.B.S.Oliveira ${ }^{1}$ \\ 1 Programa de Pós-Graduação em Modelagem Computacional, Universidade Estadual de Santa Cruz, 45662-900, \\ Ilhéus-BA, Brasil \\ 2 Doutorado em Desenvolvimento e Meio Ambiente da Associação Plena em Rede, Universidade Estadual de \\ Santa Cruz, 45662-900, Ilhéus-BA, Brasil \\ 3 Departamento de Ciências Agrárias e Ambientais, Universidade Estadual de Santa Cruz, 45662-900, Ilhéus-BA, \\ Brasil
}

\author{
*elfribeiro@uesc.br
}

\section{RESUMO}

Considerando as atuais condições dos combustíveis, o presente trabalho tem como objetivo avaliar o balanço energético e emissão de GEE do biodiesel a partir do óleo de pinhão manso, considerando o dinamismo presente na cadeia produtiva. Para isso, após a criação do esboço dinâmico, com base da ideia linear, o sistema foi divido em subsistemas e os dados foram analisados por intermédio das bases de dados Ecoinvent e PAS. Feito isso, as atividades de cada subsistema foram elencadas e tiveram informações relevantes para obtenção dos resultados pesquisadas. Os resultados reforçam a potencialidade do biodiesel de pinhão manso, ilustrado pela baixa emissão se comparado ao biodiesel de soja e mamona. Quanto ao balanço energético, o pinhão manso tem relação favorável com relação à mamona e colza, com desvantagem se comparado à soja. Desse modo, o pinhão manso vem mostrando interessantes resultados se comparado com algumas alternativas, e como se trata de uma substituição inevitável, dada à diminuição da oferta do petróleo, essa oleaginosa se mostrar cada vez mais promissora.

PALAVRAS-CHAVE: sistemas dinâmicos, viabilidade energética, biodiesel, cadeia produtiva.

\section{ENERGY RELATIONSHIP AND CARBON FOOTPRINT OF JATROPHA BIODIESEL: ANALYSIS FROM A THE DYNAMIC PERSPECTIVE}

\begin{abstract}
Considering the current conditions of fuel, the objective of this study was to evaluate the energy balance and Carbon Footprint of biodiesel from jatropha oil, considering the dynamism of the production chain. For this, after the creation of the dynamic outline, based on the linear idea, the system is divided into subsystems and the data were analyzed by means of Ecoinvent and PAS databases. Then, the activities of each subsystem were listed and had relevant information to obtain the search results. The results reinforce the potential of Jatropha
\end{abstract}

biodiesel, illustrated by low emission compared to soy and castor bean biodiesel. As for the energy balance, Jatropha has a favorable relationship with respect to castor and rapeseed, with disadvantage compared to soybeans. Thus, the jatropha has shown interesting results compared with some alternative, and as it is an inevitable replacement, given the decline in oil supply, this oilseed prove increasingly promising.

KEYWORDS: dynamic system, energy viability, biofuel, productive chain. 


\section{INTRODUÇÃO}

O crescente aumento na demanda mundial de biocombustíveis, em consequência dos planos e metas de diversos países, tem impulsionado estudos acerca da produção de alternativas viáveis. As principais vantagens obtidas a partir da substituição de combustíveis fósseis por biocombustíveis são os argumentos fundamentais para a produção e utilização dessa promissora alternativa. $\mathrm{O}$ Balanço Energético (BE), a emissão de Gases do Efeito Estufa (GEE), a produção de biomassa e a área utilizada são tidos como fatores fundamentais para análises envolvendo a produção de biocombustíveis (como bioetanol, biogás e biodiesel), que tem sido vistos como uma factível alternativa aos de origem fóssil (Cherubiniet al., 2009; Souza et al., 2010).

Dentre as vantagens do uso do biodiesel, como o provável substituto do diesel, podemos destacar: (i) fonte de energia renovável; (ii) sua combustão não contribui para a emissão de $\mathrm{CO}_{2}$; (iii) elevado ponto de fulgor $\left(150{ }^{\circ} \mathrm{C}\right)$; (iv) excelente lubricidade, aumentando a vida útil do motor; e (v) produção total mais barata do que a do petróleo (Carvalho et al., 2012).

No Brasil diversos tipos de oleaginosas podem ser utilizadas na produção de biodiesel. As mais populares são a soja, o girassol, algodão, dendê, amendoim e a mamona, além do pinhãomanso (Jatropha curcas L.) (Arruda et al., 2004). Este último é uma espécie exótica, que não foi completamente domesticada e somente nas últimas décadas começou a ter alguns aspectos pesquisados (Sato et al., 2009; Matsuura et al., 2010). A Tabela 1 apresenta características das principais oleaginosas com potencial para produção de biodiesel.

Tabela 1 - Características das principais oleaginosas utilizadas para produção de biodiesel

\begin{tabular}{|l|l|l|l|}
\hline Oleaginosa & Nome Científico & Teor de óleo $(\%)$ & Rendimento $\left(\mathbf{t}\right.$ óleo ha $\left.\mathbf{~}^{\mathbf{1}}\right)$ \\
\hline Dendê & Elaeis guineensis L. & 22 & $3,0-6,0$ \\
\hline Babaçu & Orbignya phalerata Mart. & 66 & $0,1-0,3$ \\
\hline Girassol & Helianthus annuus L. & $38-48$ & $0,5-1,9$ \\
\hline Colza & Brassica napus L. & $40-48$ & $0,5-0,9$ \\
\hline Mamona & Ricinus communis L. & $45-50$ & $0,5-0,9$ \\
\hline Soja & Glycine max L. Merrill & 18 & $0,2-0,4$ \\
\hline Algodão & Gossypum hirsutum L. & 15 & $0,1-0,2$ \\
\hline Amendoim & Arachis hypogea L. & $40-43$ & $0,6-0,8$ \\
\hline Pinhão Manso & Jatropha curcas L. & 34 & 0,44 \\
\hline
\end{tabular}

Fonte: BRASIL (2006).

Alguns motivos do potencial do pinhão-manso são: (i) capacidade produtiva da planta acima de quarenta anos (iniciada já a partir do primeiro) e com pico de produção a partir do quarto (Laviola \& Dias, 2008); (ii) adaptável em áreas marginais e degradadas (que não estão aptas para cultivos alimentares) (Matsuura et al., 2010; Mattos, 2001); (iii) forte resistência à seca, bem como variações de solo e clima (Arruda et al., 2004); (iv) perene e de fácil cultivo (Arruda et al., 2004) e (v) produtividade média de óleo de 2 t/ha (Laviola \& Dias, 2008).

A discussão acerca da produção e uso de biocombustíveis deve considerar, dentre outros fatores o monitoramento de toda a cadeia de produção, os conflitos internos ao sistema e a emissão de poluentes. Esta avaliação é feita utilizando a metodologia da Avaliação do Ciclo de Vida (ACV), metodologia utilizada na quantificação dos impactos ambientais de determinado processo durante toda sua vida útil, por meio da avaliação e cálculo de toda energia, materiais consumidos e emissões 
geradas (Almeida Neto et al., 2004). Dentre as possibilidades de abordagem, destaca-se "do berço ao túmulo", (isto é, da extração dos recursos na natureza até o descarte final) (Hinzet al., 2007). Essa metodologia segue o padrão definido na ISO 14040 e ISO 14044. No entanto, apesar de bem formulada, nem sempre as implicações causadas pelas interações do homem possuem relações estáticas e diretas, factíveis de observação (Arbaultet al., 2014).

Dessa forma, para que um modelo seja melhor compreendido, se faz necessária a introdução de uma ótica dinâmica, considerando as articulações entre os subsistemas ao longo do tempo. E considerando que a ACV utiliza dados constantes, a modelagem pode ser feita conforme os Sistemas Dinâmicos (SD) (Arbaultet al., 2014), técnica desenvolvida por Jay W. Forrester para, inicialmente, facilitar o entendimento nos processos produtivos e que, ao longo do tempo, tornou-se uma ferramenta importante para tomada de decisões. SD pode ser definido como uma metodologia de análise de determinado processo ao longo do tempo (Ferreira \& Borenstein, 2007), em outras palavras, os SD estudam sistemas suscetíveis às influências externas (Faleiros \& Youneyama, 2002).

A utilização de combustíveis fósseis gera emissões ao longo de seu ciclo de vida, as quais são responsáveis por uma das mais relevantes categorias de impacto ambiental: o aquecimento global, devido ao efeito estufa. Esta categoria de impacto está relacionada à emissão de GEE (EPE, 2007).

GEE pode ser entendido como substâncias que retém parte da radiação infravermelha, mantendo, desse modo, a Terra aquecida. Geralmente, é produzida pelos seres humanos em diversas atividades, principalmente pela queima de combustíveis não-renováveis, atividades industriais e queimadas. No entanto, a produção de biodiesel também contribui para a emissão de GEE ao longo do seu ciclo de vida, seja na fase agrícola (plantação da lavoura, colheita, etc.) como na fase industrial (transesterificação), justificando a importância da sua avaliação (EPE, 2007; Mattos, 2001).

Vale destacar que a emissão de GEE varia de acordo com a matéria prima, com a(s) tecnologia(s) utilizada(s), com os limites do sistema, com a área de estudo e com o período de tempo considerado. Além disso, geralmente, a produção de biocombustíveis resulta na geração de co-produtos, que podem substituir alguns produtos convencionais, fornecendo benefícios econômicos e ambientais para a cadeia produtiva do biocombustível (Cherubini et al., 2009).

Parâmetro importante na análise da viabilidade, o BE estabelece os fluxos de energia, contabilizando a proporção entre o total de energia empregada na produção (energia consumida no processo de produção: input) e o total de energia produzida (energia disponibilizada pelo combustível produzido: output) em todas as fases da produção, visando verificar a eficiência da relação entre a entrada e saída de energia do processo de produção dos biocombustíveis. Assim, apresenta um indicador de viabilidade energética para os biocombustíveis, a depender da quantidade de energia necessária e produzida, através das relações de diferença, output-input (O-I) e razão, output/input (O/I) (Almeida Neto et al., 2004; EPE, 2007).

Tendo em vista o objetivo do BE, diversos modos de quantificar o resultado da relação output/input são encontrados na literatura, sendo a Demanda de Energia Acumulada (DEA) uma das alternativas mais utilizadas, estando associada à abordagem em ciclo de vida. A DEA é determinada pela razão entre o somatório de todas as energias de entradas em todos os processos do sistema, sobre a energia contida nos produtos do sistema (Fukurozaki, 2011).

Diante do exposto, o objetivo deste trabalho foi avaliar o desempenho ambiental do biodiesel a partir do óleo de pinhão manso, quantificando aspectos sobre o BE e o GEE da cadeia 
produtiva e comparando sua viabilidade com outras oleaginosas produtoras de biodiesel, considerando o dinamismo existente da cadeia produtiva.

\section{MATERIAIS E MÉTODOS}

Na realização do estudo, os dados primários foram coletados em uma lavoura de sequeiro de uma propriedade rural do município de Vitória da Conquista, Região Sudoeste do estado da Bahia, Brasil. No local, a precipitação média anual é de $732 \mathrm{~mm}$, temperatura média de $20,2{ }^{\circ} \mathrm{C}$ e temperatura mínima de $11^{\circ} \mathrm{C}$, com ocorrência de neblina no inverno. Com latitude de $15,95^{\circ}$ sul, longitude de $40,88^{\circ}$ oeste e altitude de 839 metros acima do nível do mar. A plantação utiliza uma área agrícola de cinco hectares.

O período do ciclo produtivo foi sensibilizado para que fosse possível a obtenção de diferentes cenários: (i) no primeiro cenário foi considerado um ciclo de vida de quatro anos, pois é quando a lavoura alcança (inicialmente) o ápice da produção; (ii) num segundo cenário consideramos um período de quinze anos porque é o tempo médio produtivo e, por fim (iii) no terceiro cenário supomos um ciclo de vida de quarenta anos, pois é o período pelo qual a lavoura obtém produção máxima.

Os fatores de alocação de emissão utilizados (que indica quanto o material emite de $\mathrm{CO}_{2}$ ) e a DEA utilizada no cálculo do BE (que representa o total de energia investida pelo material) de cada produto e/ou processo foram obtidos a partir das bases de dados Ecoinvent 2.2 e PAS 2050, utilizando os softwares SimaPro e Umberto for Carbon Footprint no seu processamento.

A unidade funcional, utilizada neste estudo para "medir" o desempenho das saídas funcionais do sistema (constituindo referência para a qual as entradas e as saídas são relacionadas), foi uma tonelada de biodiesel de pinhão manso. A fronteira do sistema definida para este estudo foi do viveiro de mudas até a produção do biodiesel e pode ser vista na Figura 1.

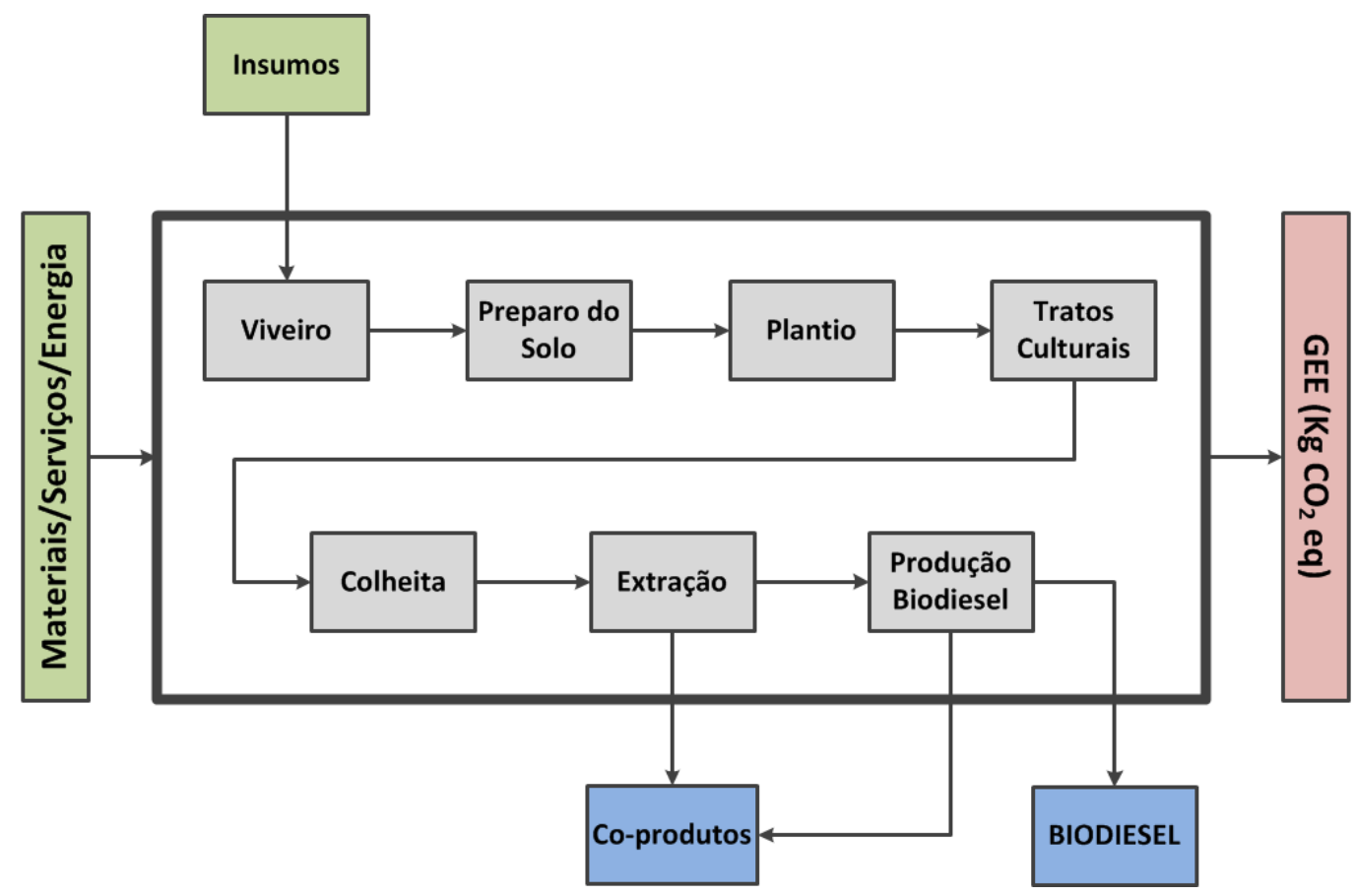

Figura 1 - Definição da fronteira do sistema da produção de biodiesel.

Fonte: Elaborado pelos autores (2015). 
De posse dos limites, um esboço dinâmico (Figura 2) foi desenvolvido para sinalizar a ideia das inter-relações entre os subsistemas existentes ("tmp" indica que podem ocorrer acréscimos de tempo que independe do sistema modelado).

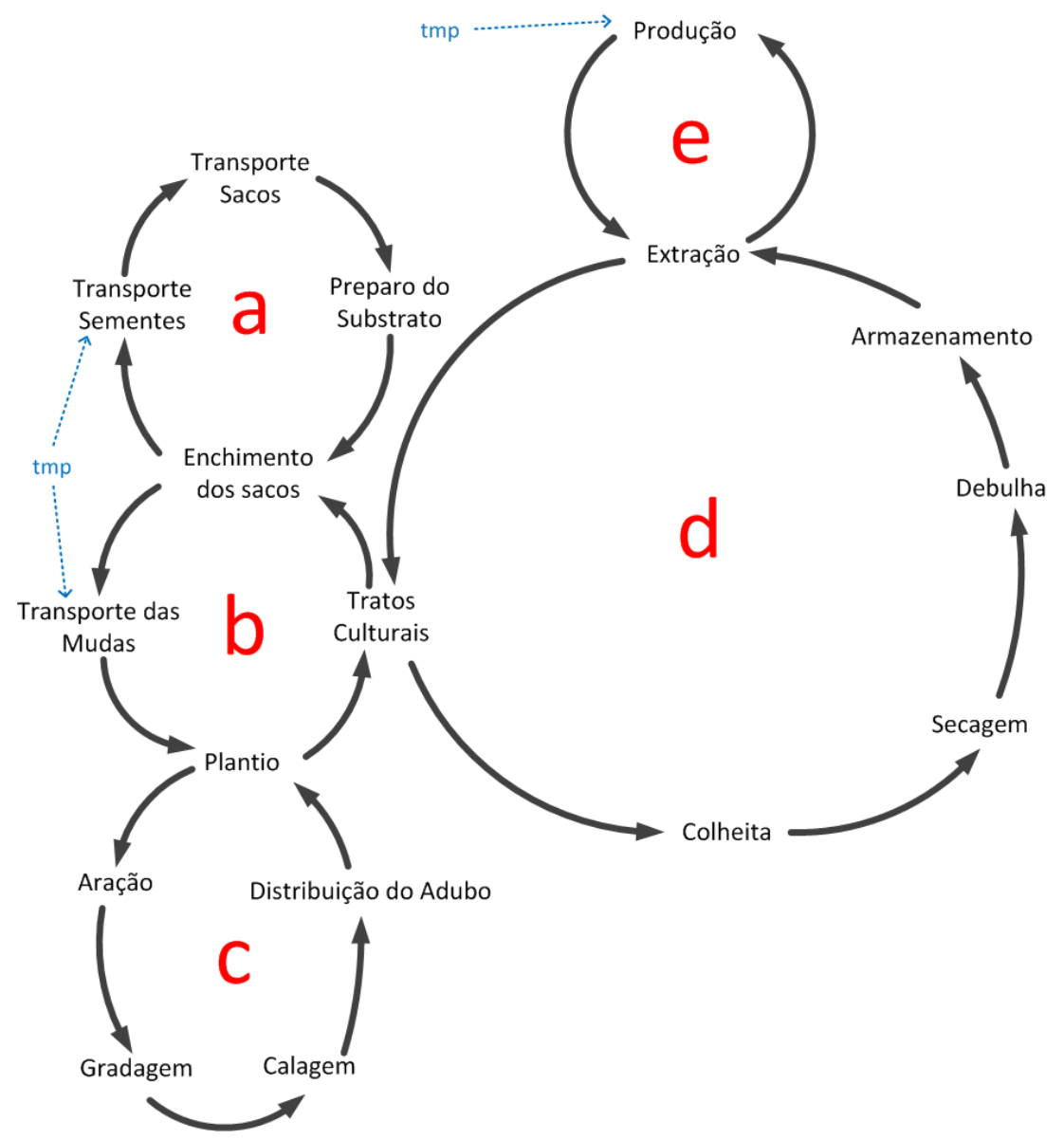

Figura 2 - Esboço dinâmico do processo. Subsistemas existentes: (a) Preparo das mudas; (b) Plantio com tratamento; (c) Preparo do solo; (d) Etapas pós-plantio; e (e) Produção de Biodiesel.

Fonte: Elaborado pelos autores (2015).

\subsection{Emissão dos Gases do Efeito Estufa (GEE)}

Nessa etapa foi utilizada os princípios da abordagem e, ciclo de vida, conforme especificada pelas normas ISO 14040 e ISO 14044.

Como mostra a Figura 2, a produção foi dividida em subsistemas: (a) Preparo das mudas; (b) Plantio com tratamento; (c) Preparo do solo; (d) Etapas pós-plantio e (e) Produção de Biodiesel. Para cada subsistema, foram elencadas as atividades necessárias para sua realização, bem como, sua respectiva emissão de $\mathrm{CO}_{2}$. Os dados podem ser visualizados na Figura 3. 


\begin{tabular}{|c|c|c|c|}
\hline Subsitema & Emissão & Subsitema & Emissão \\
\hline Preparo das mudas & 725,81 & Prepado do solo & 2456,30 \\
\hline Transporte dos sacos & 44,58 & Plantio & 1214,30 \\
\hline Transporte das sementes & 1,28 & Aração & 500,00 \\
\hline Preparo do Substrato & 658,92 & Gradagem & 314,00 \\
\hline \multirow[t]{2}{*}{ Enchimento dos Sacos } & 21,04 & Calagem & 314,00 \\
\hline & & Distribuição do Adubo & 114,00 \\
\hline Plantio com tratamento & 62775,28 & & \\
\hline Enchimento dos Sacos & 21,04 & & \\
\hline Transporte das Mudas & 0,00 & Etapas pós-plantio & 65852,09 \\
\hline Plantio & 1214,30 & Tratos Culturais & 61539,94 \\
\hline \multirow[t]{2}{*}{ Tratos Culturais } & 61539,94 & Colheita & 0,00 \\
\hline & & Secagem & 408,72 \\
\hline Produção de Biodiesel & 22690,84 & Debulha & 0,00 \\
\hline Extração & 2656,50 & Armazenamento & 1246,93 \\
\hline Produção & 20034,34 & Extração & 2656,50 \\
\hline
\end{tabular}

Figura 3 - Inventário detalhado das etapas da cadeia produtiva com base no esboço dinâmico, com respectivas emissões (em $\mathrm{kg} \mathrm{CO}_{2}$-eq), para um ciclo de vida de quinze anos.

Fonte: Elaborado pelos autores (2015).

Com o agrupamento das emissões, para cada cenário, podemos visualizar as emissões pertinentes a cada processo. Assim, a emissão em cada cenário (para cada tonelada de biodiesel) é dada pela fórmula:

$$
\frac{\sum e\left(m_{i}\right)}{\text { ProdTotal }}
$$

Onde: $e\left(m_{i}\right)$ é a emissão do $i$-ésimo material; e ProdTotal é a massa total em de toneladas de biodiesel, produzida sob as características do cenário.

O processo de extração do óleo de pinhão manso, considerado nessa avaliação, permite um rendimento de aproximadamente $34 \%$ de óleo bruto, considerando que foi adotada a extração mecânica no processo produtivo (Carvalho \& Costa, 2009).

\subsection{Balanço Energético (BE)}

Para fins de comparação, optou-se por desconsiderar nessa etapa, a fase da produção do biodiesel, isto é, calculou-se até a etapa da extração do óleo. Isso se justifica porque os resultados obtidos independem da rota de produção adotada na produção do biodiesel, uma vez que o determinante da viabilidade neste caso é a matéria-prima (Almeida Neto et al., 2004).

Ainda conforme o mesmo autor, os inputs do sistema podem ser divididos em:

a) Energia Direta: é a energia (fóssil, eletricidades, etc.) consumida durante a cadeia produtiva. Calculamos com base no Poder Calorífico Inferior (PCI).

b) Energia Indireta: é a energia consumida por intermédio dos insumos, máquinas, etc. Avaliamos com base na Demanda de Energia Acumulada (DEA), que indica o quanto de energia é necessário para a produção de uma unidade de um determinado produto.

c) Energia de Transporte: é a energia dispensada para o transporte, tendo como base para o cálculo a distância percorrida e o tipo de transporte utilizado.

Ainda de acordo com o mesmo autor, o output é calculado com base na energia disponível nos co-produtos e no produto final. Obtivemos o BE realizando o somatório de todos os inputs e 
relacionando-o com o respectivo output, para cada cenário. Nesse caso, o output é calculado com base na energia liberada na combustão do próprio óleo, quantificado pelo seu PCI (que indica a energia emitida pela sua combustão).

Desse modo, o BE é dado pelas fórmulas:

$$
\frac{\sum \operatorname{dea}\left(I_{i}\right)}{\operatorname{pci}(O)}
$$

e por

$$
\sum \operatorname{dea}\left(I_{i}\right)-p c i(O)
$$

Onde: $\operatorname{dea}\left(I_{i}\right)$ é a DEA do $i$-ésimo material ou serviço; e $p c i(O)$ é o PCI do(s) output( $(s)$.

Nesse estudo, consideramos o PCI do óleo de pinhão manso com valor de 39,5 MJ/kg (RAHMANet al., 2010).

Vale destacar que a Equação 2 foi utilizada no cálculo da relação I/O, e a Equação 3 para a estimativa de I-O.

\section{RESULTADOS E DISCUSSÃO}

Levando em consideração os três cenários descritos, os resultados estão expostos a seguir. Foi adotada a seguinte nomenclatura para os cenários:

a) $\mathrm{C}_{1}$ : cenário para um ciclo de vida de quatro anos;

b) $\mathrm{C}_{2}$ : cenário para um ciclo de vida de quinze anos;

c) $\mathrm{C}_{3}$ : cenário para um ciclo de vida de quarenta anos.

\subsection{Emissão dos Gases do Efeito Estufa (GEE)}

A Tabela 2, abaixo, ilustra as emissões de GEE para cada um dos cenários propostos.

Tabela 2 - Emissão de GEE (em Kg $\mathrm{CO}_{2}$-eq/t) para cada cenário proposto

\begin{tabular}{|l|r|}
\hline Cenário & \multicolumn{1}{|c|}{ Emissão } \\
\hline$C_{1}$ & 1051,25 \\
\hline$C_{2}$ & 622,82 \\
\hline$C_{3}$ & 580,14 \\
\hline
\end{tabular}

Fonte: Elaborado pelos autores (2015).

Conforme a Figura 4, podemos visualizar o comparativo das emissões de GEE das seguintes oleaginosas utilizadas para produção de biodiesel: soja e mamona. A figura 4 compara, supondo o cenário $\left(\mathrm{C}_{2}\right)$, por ter o mesmo tempo do ciclo de vida utilizado nos estudos equivalentes (para as diferentes matérias-primas). Nesse caso, o pinhão manso apresentou o melhor desempenho, com a menor emissão de GEE. 


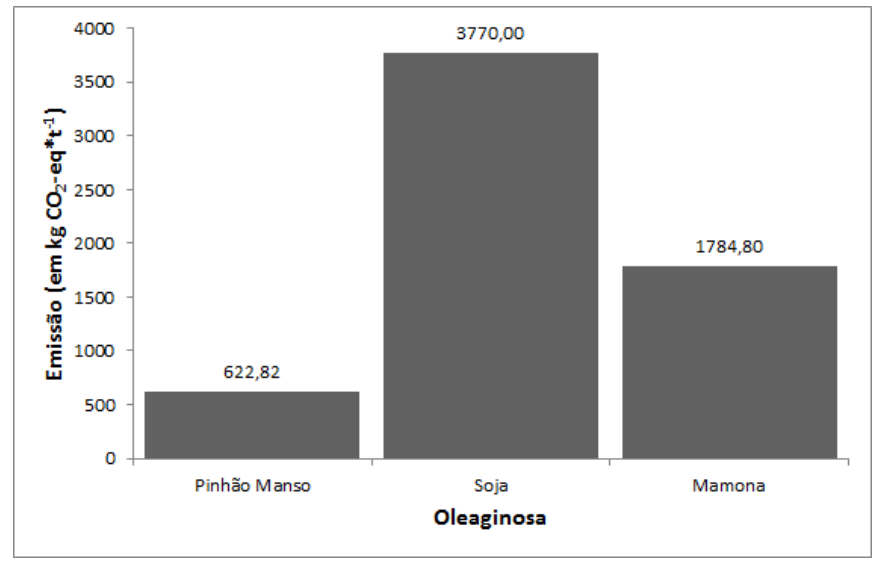

Figura 4 - Comparativo para três oleaginosas da emissão de GEE por tonelada de Biodiesel.

Fonte: Elaborado pelos autores (2015).

Na Figura 5, que detalha o processo produtivo, é possível visualizar as emissões individuais de cada subsistema, desse modo pode-se atribuir, a cada subsistema, sua interferência na emissão total do sistema. Com base nesta premissa, identificam-se os aspectos críticos do modelo, possibilitando identificar potenciais de melhoria e aprimoramento do processo produtivo, visando a redução das emissões.

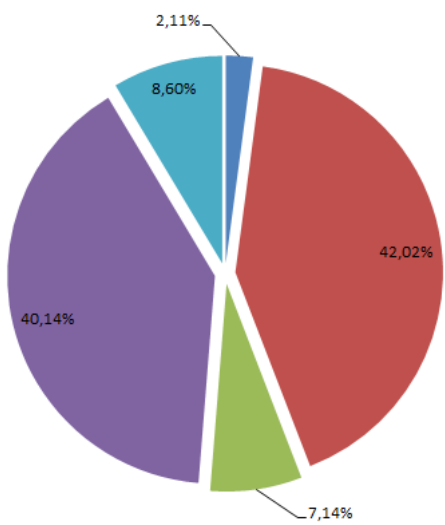

(a) Emissão detalhada de $\mathrm{C}_{1}$

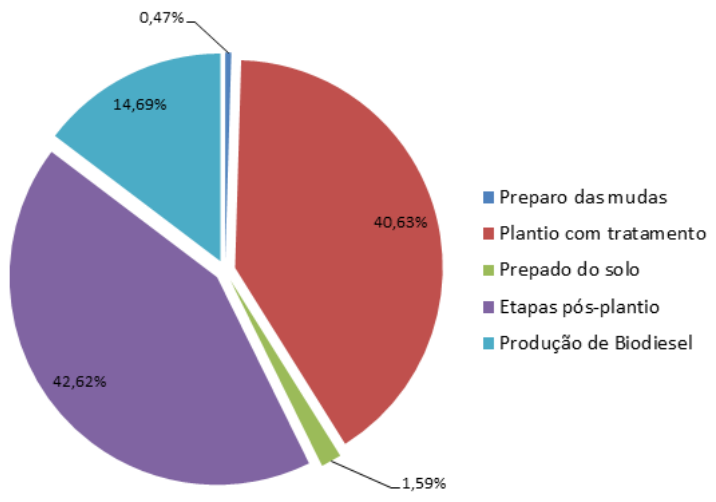

(b) Emissão detalhada de $\mathrm{C}_{2}$

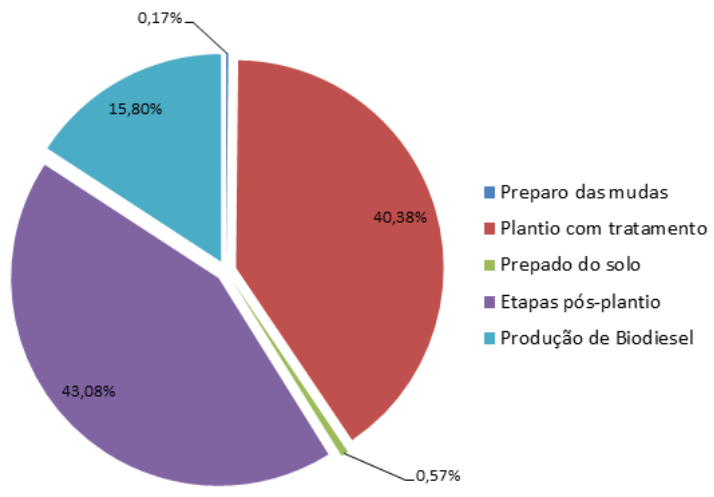

(c) Emissão detalhada de $\mathrm{C}_{3}$

Figura 5 - Emissões por etapa do processo produtivo para todos os cenários. 
Fonte: Elaborado pelos autores (2015).

É possível visualizar que, nos cenários de maiores intervalos de tempo $\left(\mathrm{C}_{2}\right.$ e $\left.\mathrm{C}_{3}\right)$, o processo responsável pela maior emissão foram as etapas pós-plantio. Isso ocorre pela grande quantidade de fertilizantes utilizados, como podemos ver na Figura 6 (apenas para $\mathrm{C}_{2}$ ). A maior emissão da etapa é causada pelo Superfosfato Simples ( 46\%), seguido pelo fertilizante composto 20-00-20 ( 34\%).

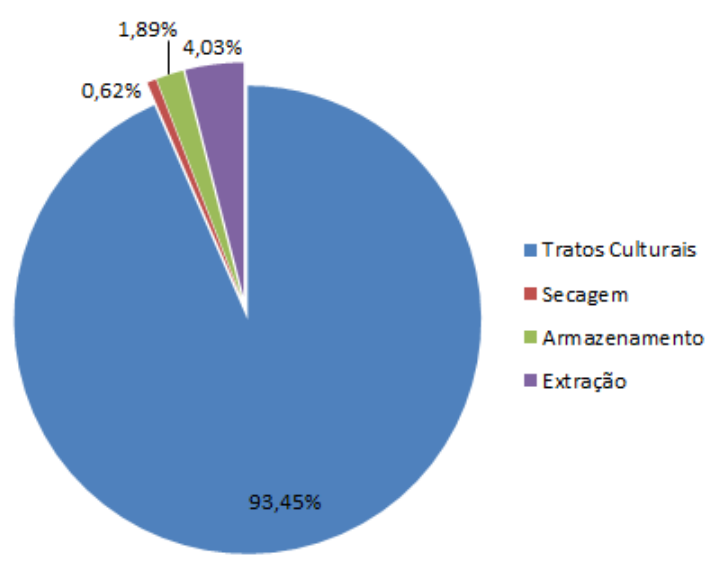

Figura 6 - Percentuais de emissão por etapas no pós-plantio para cenário $\mathbf{C}_{2}$. Fonte: Elaborado pelos autores (2015).

\subsection{Balanço Energético (BE)}

Assim como o indicador anterior, o BE do Pinhão Manso também mostrou uma relação comparativa favorável, considerando as matérias-primas usuais para a produção de biodiesel.

A Figura 7 ilustra um comparativo da relação $\mathrm{O} / \mathrm{I}$ do pinhão manso com relação à mamona, soja (produzida nos EUA) e colza (produzida na Europa). A energia investida no processo produtivo e a contida no óleo de pinhão manso foram maiores do que valores encontrados para mamona e colza. No entanto, foi menor que os valores encontrados para a soja.

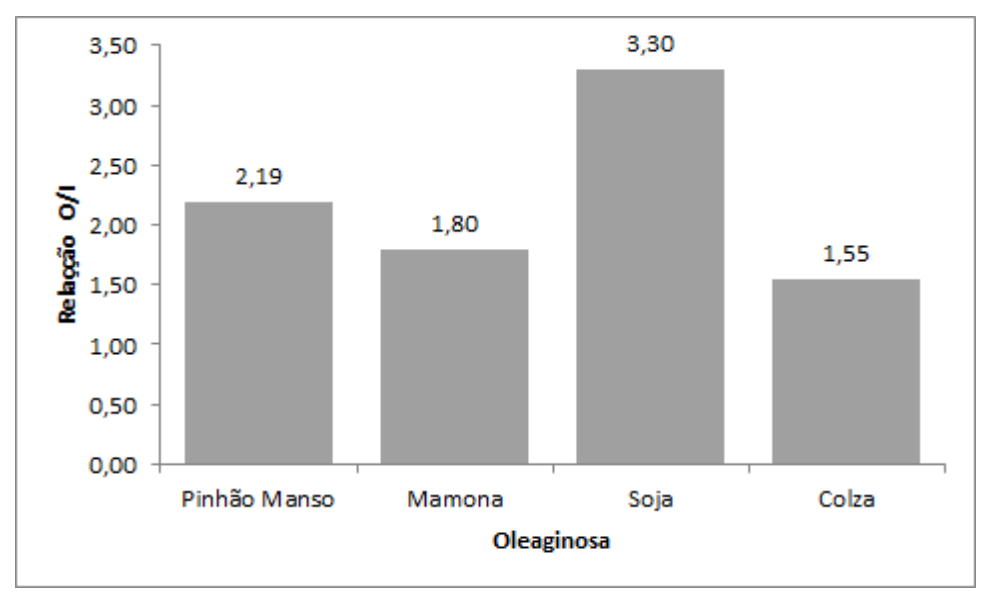

Figura 7 - Comparativo da relação O/I.

Fonte: Elaborado pelos autores (2015). 
Um comparativo da energia líquida produzida por hectare $\left(\mathrm{GJ} \mathrm{ha}^{-1}\right)$ do óleo de pinhão manso pode ser vista na Figura 8. Nesse caso, os valores foram maiores do que aqueles encontrados para a mamona e para a soja, reforçando a potencialidade da oleaginosa.

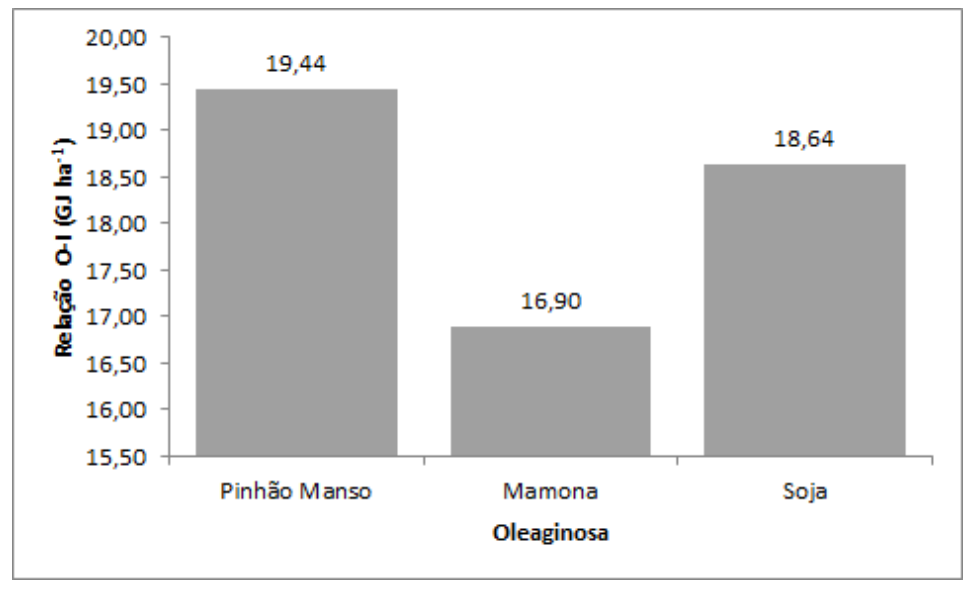

Figura 8 - Comparativo da relação O-I.

Fonte: Elaborado pelos autores (2015).

\section{CONSIDERAÇÕES FINAIS}

Mesmo considerando que o pinhão manso (Jathropa curcas L.) ainda não esteja completamente domesticado e com parâmetros de produção agrícola definidos, seu potencial aponta para ser uma oleaginosa promissora no cenário futuro da produção de biocombustíveis. Dentre os motivos, podemos destacar: (i) a alta capacidade produtiva; (ii) o elevado teor de óleo; (iii) a baixa emissão de GEE; (iv) a favorável relação energética; (v) a pouca exigência de solo e clima e (vi) o fato de não concorrer com a alimentação humana e animal.

Pelos resultados, observa-se que o tempo considerado como ciclo de vida da lavoura foi inversamente proporcional à emissão de GEE, visto que alguns consumos e emissões (como no caso da etapa de implantação) ocorrem uma única vez, o que tende a ser amortizado no decorrer do tempo. Outro fato que beneficia esse resultado é a elevada produtividade de óleo da oleaginosa, que tende a compensar as elevadas emissões que ocorrerão ao longo do tempo.

Por fim, destaca-se a significativa contribuição que os biocombustíveis, em especial o biodiesel, poderão fornecer no médio e longo prazo, para impulsionar a substituição dos combustíveis fósseis não renováveis na matriz energética, considerando o avanço das políticas de mitigação das mudanças climáticas.

\section{AGRADECIMENTOS}

Os autores agradecem todo suporte na realização dessa pesquisa, em especial à FAPESB, ao PPGMC e à UESC.

\section{REFERÊNCIAS}

ALMEIDA NETO, J.A. de; CRUZ, R.S.; ALVES, J.M.; PIRES, M.M.; ROBRA, S.; PARENTE JR, E. Balanço energético de ésteres metílicos e etílicos de óleo de mamona. In: CONGRESSO BRASILEIRO DE MAMONA ENERGIA E SUSTENTABILIDADE, 1., 2004, Campina Grande. Anais... Campina Grande: EMBRAPA, 2004. 
ARBAULT, D.; RIVIÈRE, M.; RUGANI, B.; BENETTO, E; TIRUTA-BARNA, L. Integrated earth system dynamic modeling for Life Cycle impact Assessment of ecosystem services. Science of The Total Environment, v.472, n.1, p. 262-272, 2014.

ARRUDA, F.P.; BELTRÃO, N.E.M.B.; ANDRADE, A.P.; PEREIRA, W.E.; SEVERINO, L.S. Cultivo de pinhão manso (Jatropha curca L.) como alternativa para o semi-árido nordestino. Revista Brasileira de Olericultura Fibrose, Campina Grande, v.8, n.1, p. 789-799,2004.

BRASIL - MINISTÉRIO DA CIÊNCIA E TECNOLOGIA. Plano Nacional de Agroenergia. Brasília: EMBRAPA, 2006.

CARVALHO, H.M.; RIBEIRO, A.B. Biodiesel: Vantagens e desvantagens numa comparação com o diesel convencional. Bolsista de Valor: Revista de divulgação do Projeto Universidade Petrobras e IF Fluminense, Rio de Janeiro, v.2, n.1, p. 49-53, 2012.

CARVAlHO, M.G.; COSTA, A. Avaliação do Valor Fertilizante de Tortas Oleaginosas para Adubação Orgânica. Revista Brasileira de Agroecologia, v.4, n.2, 2009.

CHERUBINI, F.; BIRD, N.D.; COWIE, A.; JUNGMEIER, G.; SCHLAMADINGER, B.; WOESS-GALLASCHA S. Energy and greenhouse gas based LCA of biofuel and bioenergy systems: Key issues, ranges and recommendations. Resources, Conservation and Recycling. v.53, n.1. p. 434-447, 2009.

EPE - Empresa de Pesquisa Energética. Potencial de redução de emissões de $\mathrm{CO}_{2}$ em projetos de produção e uso de biocombustíveis.Rio de Janeiro: Fundação Brasileira para o Desenvolvimento Sustentável, 2007.

FALEIROS, A.C.; YONEYAMA, T. Teoria matemática de sistemas, São Paulo: Arte e Ciência, 2002.

FERREIRA, L.; BORENSTEIN, D. Análise da viabilidade da produção de biodiesel a partir da soja no Brasil. In: SIMPÓSIO BRASILEIRO DE PESQUISA OPERACIONAL, 39., 2007, Fortaleza. Anais... Fortaleza: SOBRAPO, 2007.

FUKUROZAKI S.H. Avaliação do Ciclo de Vida de Potenciais Rotas de Produção de Hidrogênio: Estudos dos Sistemas de Gaseificação da Biomassa e de Energia Solar Fotovoltaica. 2011. Tese (Doutorado em Ciências) Universidade de São Paulo, São Paulo. 2011.

HINZ, R.T.P.; VALENTINA L.V.D.; FRANCO, A.C.; Monitorando o desempenho ambiental das organizações através da produção mais limpa ou pela Avaliação do Ciclo de Vida. Revista Produção online. v.7, n.3, 2007.

LAVIOLA, B.G.; DIAS, L.A.S. Teor e acúmulo de nutrientes em folhas e frutos de pinhão-manso. Revista Brasileira de Ciência do Solo. v.32, n.1, p. 1969-1975, 2008.

MATSUURA, M.I.S.F.; SILVA, G.A.; KULAY, L.A.; LAVIOLA, B.G. Inventário do Ciclo de Vida do pinhão manso destinado à produção de Biodiesel. In: CONGRESSO BRASILEIRO EM GESTÃO DE CICLO DE VIDA EM PRODUTOS E SERVIÇOS, 2., Florianópolis. Anais... Florianópolis, 2010.

MATTOS, L.B.R. A importância do setor de transportes na emissão de Gases do Efeito Estufa - o caso do município do Rio de Janeiro. 2001. Dissertação (Mestrado em Engenharia) - Instituto Alberto Luiz Coimbra de PósGraduação e Pesquisa de Engenharia, Universidade Federal do Rio de Janeiro, Rio de Janeiro. 2001.

RAHMAN K.M.; MASHUD, M.; ROKNUZZAMAN, M.; GALIB, A.A. Biodiesel from Jatrobha Oil an as Alternative Fuel for Diesel Engine. International Journal of Mechanical \& Mechatronics Engineering, v.17, n.3, p. 1-6, 2010.

SATO, M.; BUENO, O.C.; ESPERANCINI, M.S.T.; FRIGO, E.P. A cultura do pinhão manso (Jatropha curcas L.): uso para fins combustíveis e descrição agronômica. Revista Varia Scientia, v.7, n.13, p. 47-62, 2009.

SOUZA, S.P.S.; PACCA, S.; ÁVILLA, M.T.; BORGES, J.L.B. Greenhouse gas emissions and energy balance of palm oil biofuel. Renewable Energy. v.35, n.1.p. 2552-2561, 2010. 